\title{
A Computational Model of Emotions for the Simulation of Human Emotional Dynamics in Emergency Situations
}

\author{
Mouna Belhaj, Fahem Kebair, and Lamjed Ben Said
}

\begin{abstract}
Emotions have a considerable effect on human behaviors and cognitive processes, especially during crisis situations. Emotion modeling is therefore a key solution to generate realistic social simulations in crisis situations. In this context, we intend to model human emotional dynamics and to study their effect on individual and collective behaviors during emergency situations. In this paper, we focus on the first part of this research work which consists in the modeling of emotion generation in emergency situations. Thus, we provide first a modeling of the disaster space in a rescue simulation context. Then, we propose a computational model of the generated human emotions, basing on the emergency environment. This model uses the appraisal theories of emotions.
\end{abstract}

Index Terms-Appraisal, emergencies, emotional dynamics, human behavior.

\section{INTRODUCTION}

Modeling human behavior in normal or emergency situations is useful in different applications including safety planning, virtual training, serious games and human crowd simulations in normal situations or during emergency evacuations [1]. Modeling human behaviors during emergencies was long covered by mathematical, physical and cellular automata models. These approaches are mainly concerned with the study of the collective aspect of human behavior. Their major drawback is that they reduce humans to particles and their complex behaviors to pre-programmed simple navigational behaviors [2], [3]. Recently, there has been an orientation to agent based modeling of human behavior with a special emphasis on psychological, social and cognitive factors in order to reproduce realistic behaviors in believable simulations. Emotions and personality are among the most important factors that modulate human behavior, particularly during emergencies. They has been therefore recognized to be necessary in simulating realistic behaviors during such situations [4].

In our research work, we aim to study the effect of emotions on the behavior of civilians in an emergency situation after a disaster. The objective is to construct acivilian human simulator where each civilian is represented by an emotional empathetic agent. This article is dedicated to the description of the emotion generation process. The role of emotions on producing realistic behaviors will be illustrated in future publications. The study of emotion consequences on

Manuscript received October 3, 2013; received January 7, 2014.

The authors are with the Higher Institute of Management of Tunis (ISG) and with the Optimization Strategies and Intelligent Information Engineering Laboratory (SOIE Lab), Le Bardo, 2000, Tunisia (e-mail: mouna.belhaj@hotmail.com, lamjed.bensaid@isg.rnu.tn). human behaviors during emergencies requires an investigation on the study of emotions at an individual and a collective level. At the individual level, emotions influence perception, decision making and action selection. At the collective level, emotions play a social role in promoting social behaviors. Indeed, collective emotions may appear through emotional contagion mechanisms. Empathetic emotions and behaviors may be modeled thus to enhance the realism of human behavior simulations. Inspired by existing computational models of appraisal, we propose a computational model of emotions for civilians in a rescue simulation context. The model we propose is based-on on the OCC model [5]. The latter is a psychological model of emotions for computational modeling.

This paper is structured as follows. First, we provide an overview on computational models of emotions and their theories with a particular interest on appraisal theories of emotions. Then, we propose a generic disaster space model on which relies the generation of emotions. Next, we describe the proposed computational model of emotions that will serve for a human behavioral simulator during emergency situations. Finally, we discuss the different perspectives that follow the present work.

\section{BACKGROUND}

\section{A. Cognitive Theories of Emotions}

The literature contains various computational models of emotions that are based on psychological theories. They are particularly founded on cognitive theories of emotions. These include, for example, the anatomical theory of Damasio [6] and the rational theory of Sloman and Croucher [7]. Although different theories of emotions exist, the majority of approaches on emotion computational models are based on appraisal theories. These are cognitive theories of emotions that include the theory of Scherer [8], theory of Lazarus \& Smith [9] and the OCC model [5] to name a few.

\section{B. The OCC Model}

The OCC model [5] was conceptualized by specialists on psycho-cognition and was designated to computational modeling. The OCC model is consequently the most popular in computational models of emotions. In cognitive appraisal theories, emotions are considered as reactions resulting out of an evaluation of the agent-environment relationship. The OCC model defines 22 pairs of emotions that represent valenced reactions to events, actions and objects.

When a change in the environment is recognized, it is evaluated using specific appraisal variables. The OCC model defines a set of appraisal variables, classified into three 
categories and associated with the sets of specific emotions that they may influence (see Table I). The first type concerns local variables that have particular effects on some emotions and no effect on others. The second categoy covers central variables. Three central variables are defined and each variable is associated with only one category of emotions. Finally, global variables are variables that may influence the generation of all emotion categories.

TABLE I: EMOTION CATEGORIES AND ASSOCIATED APPRAISAL VARIABLES BASED ON THE OCC MODEL

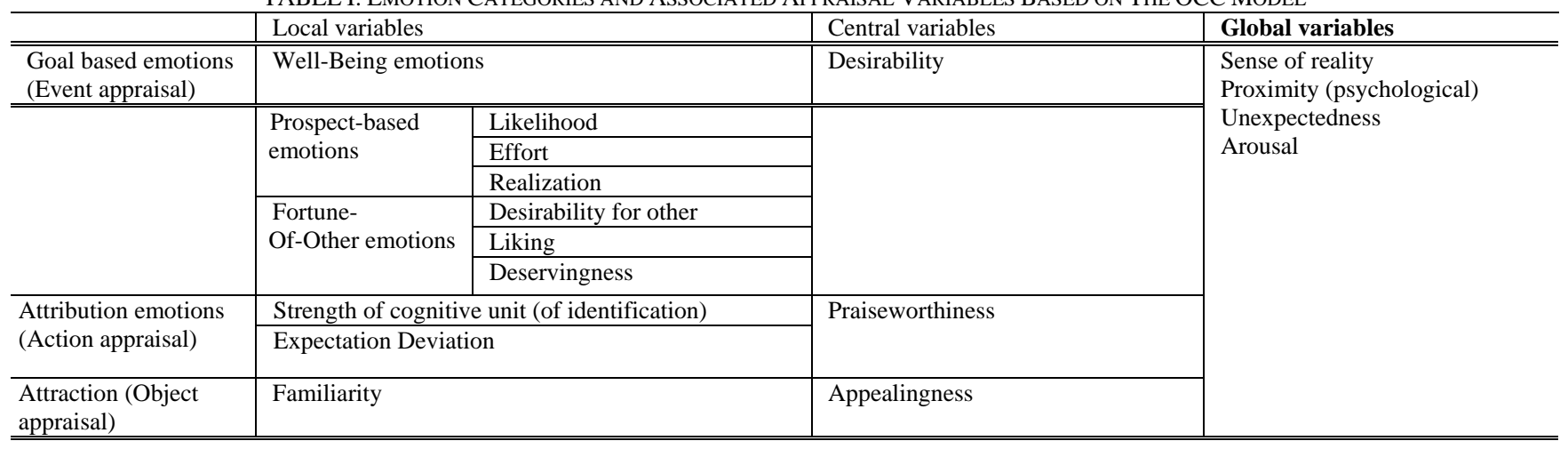

In our computational model of emotions we have used an important subset of these variables. These include five local variables: Liking, Effort, Desirability for Other, Likelihood, Realization and Familiarity and the three central variables: Desirability, Praiseworthiness and Appealingness. We have also added the Urgency variable from the theory of Scherer [8] that is an important variable in the context of emergencies. The definitions of these variables and their influence on emotions generation are described in Section IV.

\section{Computational Models of Appraisal}

Modeling human emotions is a complex and non trivial task. This is due to the inner complexity of emotion processes and the lack of a consensual theory of human emotions. There are different psychological theories that have tried to depict the different processes underlying emotion. Appraisal theories of emotion represent one of the most exploited theories for computational modeling. Some computational models of emotions were based on the theory of Scherer [8], such as the work presented in [4] that presents a computational model of emotions for crowd simulation. We can also site EMA (Emotion and Adaptation) presented in [10] as example of the works based on Lazarus theory of emotions [9]. Many researchers have chosen to build their computational models of emotions on the OCC model [5]. Examples include the FLAME (Fuzzy Logic Adaptive Model of Emotions) model of El Nasr et al. described in [11]. The FearNot model of Dias and Paiva explained in [12]. A final example is GEmA (Generic Emotional Agent) model described in [13] among others.

We opted for the OCC model to build our computational model of emotions. Indeed, the OCC model provides a comprehensive description of emotions and indications about their computation. Moreover, the use of appraisal variables is

appropriate to the quantitative aspect of our environment modeling. In fact, this will allow a smooth deduction of the appraisal variable values and then the computation of emotion values. The approach we propose differs from the existing models of appraisal on the generation of emotions, by using a different subset of appraisal variables and quite different methods to compute them. Moreover, the emotion generation process modeling is only the first step of our study of realistic behaviors during emergencies. Further steps consist on the study of the effect of empathy and emotion based interactions on behaviors. Primarily, we aim to study the effect of emotions on individual behaviors during an emergency situation. After that, we will investigate in the study of emotion based interactions and external emotional dynamics and their effects on collective behaviors.

\section{ENVIRONMENT MODEL}

The objective of our work is to study human emotional internal and external dynamics effects on individual and collective behaviors during emergencies. We intend to develop a multi agent civilian simulator. Each civilian is represented by an emotional empathetic agent. In an emergency situation simulation, agents operate in a dynamic environment. In fact, an emergency situation evolves rapidly and then requires rapid adaptation mechanisms. Emotions are modeled to provide agents with such mechanisms and then to enhance the realism of civilian behavior simulation. Empathy modeling is necessary to produce empathetic behaviors similar to those that appear in emergency situations and to study collective behaviors. In this paper we focus on a first step of the realistic behavior simulation that consists in modeling the emotion generation process.

Emotions are reactions to different aspects of the environment where a person is situated. In order to simulate emotion generation, we need to define and model this environment and depict its different entities, phenomena and mechanisms that will affect the emotional state of our agents.

The environment model we propose, is based on the conceptual model of the disaster space for emergency situation management presented in [14], [15]. In fact, one of the important goals of this approach is to model facts perceived by artificial agents in a crisis environment. These facts represent the appraisal base of our computational model from which agent emotions will be generated. Moreover, the approach defines a generic taxonomy that describes the different elements of an environment during a disaster. We conserved the same hierarchy of the conceptualized classes for the organization of the environment elements. However, we renamed two classes and reified the specification of the characteristics and methods of different classes. In our environment modeling, we are focusing on the aspects of the 
environment that may influence the emotions of civilians. Therefore we take a civilian's perspective in modeling this environment. In Fig. 1, we present a modified version of the model of the crisis environment presented in [14]. A civilian may perceive several objects in its environment. These objects are divided into two main categories. These are physical objects and immaterial objects. These objects are evaluated using the appraisal process of the OCC model in order to generate the emotions of civilians.

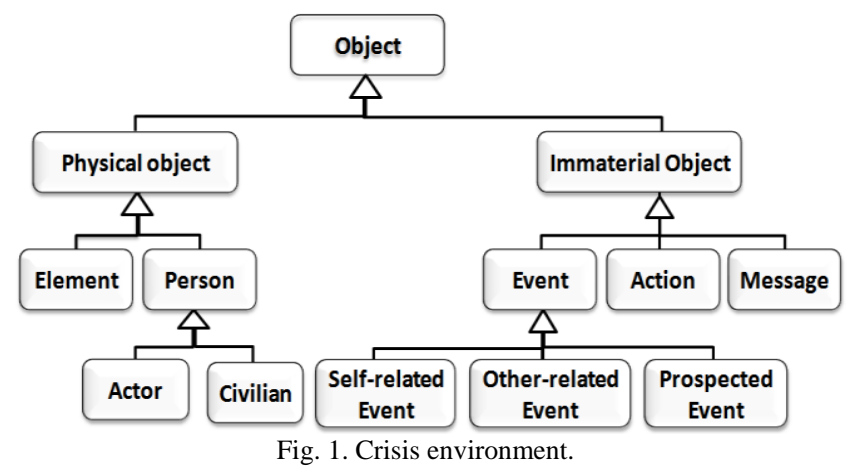

The subclasses of each category and their main properties are described in the following subsections.

\section{A. Physical Objects}

This category of objects includes elements and persons.

\section{1) Elements}

This class of objects represents the passive entities of the environment such as buildings, and roads. The properties of these elements, for example the state of a building or the damage in a road, are appraised to generate the attraction emotions (like/dislike) that result from object appraisal.

\section{2) Persons}

These are active objects that represent humans. We are particularly interested on the emotional responses of civilians to the occurred events in a disaster situation. Therefore, a decomposition of the class Person into actors and civilians is necessary in our case.

Actor class covers rescue personals. We have conserved the specification of human actors presented in [15]. Actors have a main objective that is to manage risks in order to control the evolution of the situation.

We have notably extended the design of Civilian class so that it can support the exigencies of emotion generation and update processes. In addition to the unique objective or goal defined in the original model for human civilians that was to 'Escape the risk', we have identified four other goals. These goals are: 'Keep safe', 'Get saved', 'Find a family member' and 'Help a person'. A civilian has also an emotional state, a social role, a set of standards and knowledge about himself and his environment. In Section IV, we describe the effects of emotions on the agent goals.

\section{B. Immaterial Objects}

This kind of objects allows the modeling of the environment dynamics. It includes three dynamic facts that are actions, events and messages.

\section{1) Events}

We have renamed the class 'Phenomenon' into 'Event'. A phenomenon is defined in the original model as an event that takes place in the environment such as a fire, an explosion or an inundation. Since we are interested by events influencing the emotions of civilians, we needed to define a more specific class to describe the events that concern a civilian in a disaster. We identified three categories of events that may affect directly or indirectly the states of a civilian (see Fig. 1).

Events related to a civilian; These are events that affect directly the state of a civilian by altering his physical and emotional states. These events are used to generate the so-called "Well-being" emotions of the OCC model. Events that affect directly a civilian include positive events that result on a joy emotion: Safe (self), Rescue (self), Help (self) and HealthStateUp (self). They also include negative events that result on the distress emotion: Injury (self), HealthStateDown (self) and InDanger (self). We mean by a danger any negative external event, for example perceiving a near fire, smelling a gas or hearing an explosion.

Events related to other civilians; They include events that may touch others. These events are needed to model a subset of Fortune for other emotions called empathetic emotions. These emotions have an impact on social and empathetic behaviors and on collective emotions. This category of events includes positive events that result on a Happy For empathetic emotion: Safe (other), Rescue (other) and HealthStateUp (other). They also include negative events that result on the SorryFor emotion: Injury (other), InDanger (other), HealthStateDown (other) and Death (other).

Events that a civilian prospects; A civilian acting in the crisis environment may face situations that he cannot deal with. Thus, he needs the help of others to change its embarrassing state. Therefore a third category of events, that a civilian may expect, is introduced in our modeling. These events are necessary to model prospect-based emotions of the OCC model. The prospect or the realization of a positive event results on positive emotions (hope, satisfaction): ProspectHelp (self) (from another civilian), ProspectRescue (self) and ProspectManageRisk (Element) (from an actor). When a civilian expects a negative event, he will experience negative emotions (fear, disappointment): ProspectDeath (self) and ProspectInjury (self).

\section{2) Actions}

Actions are defined as the activities that a person can carry out. We focus on civilian actions. The action results of human actors are considered as events: Rescue (self) and ManageRisk (element). A civilian appraises other civilian actions based on its standards in order to deduce attribution emotions. Actually, we consider only one standard: "helping a person in need is an action that conforms to standards": Help (person). Civilians may perform other actions as behavioral response to their current state such as Move (environment), Wait (person) and Find (Person).

\section{3) Messages}

The messages exchanged between humans represent a mean of communication. Humans can exchange information messages to inform about an event or an injured person. Messages can also be sent to ask for help. These messages may induce emotional reactions in the receiver. Sending an information or help message is also considered as a behavioral response induced by the emotional state of the agent. 


\section{EMOTION MODEL}

The emotional process may be divided into two main processes [16]. The first one is the emotion generation process. It comprises different steps including perception, appraisal variables and emotion intensities computation and emotion update. However, the second one concerns the effects that emotion can have on perception, on behavior and cognition and on memory. In the current article, we focus on the modeling of the emotion generation process.

\section{A. Perception}

According to the OCC model [5], emotions are a result of the cognitive appraisal of the events, actions and objects in the environment. Thus, perception is an important step of the emotion generation process that consists in acquiring three categories of information. The first class includes visual information. It represents a current view of the environment: actors, civilians and the other physical elements of the environment. The second category includes auditory information. It encloses information or help messages and any other noise such as explosions, collapses or ambulance sirens. The last class involves what an agent can smell in the disaster space such as smokes or gases. These percepts are processed to detect the main changes of the agent's context caused by new events or actions or changes in object aspects. The description of possible (recognizable) events, actions and objects was detailed in Section III.

\section{B. Civilian Goals}

A goal is a state that a human aims to conserve or to attain. The OCC model distinguishes three classes of goals. The first class includes Active pursuit goals. These are goals that an agent actively tries to achieve and can realize by performing some actions. The second class encloses Interest goals that represent states of the world that an agent would like to become reality but cannot do anything about pursuing them. The last type is Replenishment goals that are reactivated only on demand [5]. In the context of a major disaster, we assign to each civilian one replenishment goal (Keep safe) and four active pursuit goals (Get saved, Escape the risk, Find a family member and Help a person). The main goal of an agent in an emergency situation is to keep safe. In fact, if the civilian is physically well and the goal of keeping safe is not threatened, he may think about finding a family member or helping civilians in need. The goals of the civilian are rearranged based on physical and situational factors. If the civilian is injured or buried, his main goal will be to get saved and the other goals become of minor priority.

\section{Emotion Intensities Computation}

The significance of the perceived events, actions and object characteristics are evaluated using a set of appraisal variables. Each combination of appraisal variable values generates a distinct emotion. In this section, we present the definitions and the different parameters that influence the computation of these variables. The latter are not equivalent. A set of weights $(\alpha, \beta, \gamma$ and $\lambda)$ are therefore assigned to them. The values of these weights should be adjusted during the model implementation and tests. It can be noticed that the equations we use are not resource intensive. Moreover, the computation process is distributed since each agent performs its computations separately using the formula presented below.

\section{1) Event appraisal}

According to the OCC model, events are appraised according to their effects on the goals of a person. The three categories of events, defined in section III, are evaluated to generate three categories of goal-based emotions resulting from event appraisal.

\section{a) Well-being emotions}

Well-being emotions result from focusing attention on an event itself that is happening [5]. Events affecting the civilian's well being are evaluated using the Desirability central variable. The Desirability of an event depends on its contribution/interference to the realization of the goal of a person. The desirability of an event is generally measured through the impact of the event on the agent goals. Different methods are used in [11], [13] and [17] to compute the desirability of an event. However, they share the same main idea. The latter consists in assigning some importance values to each goal (Fuzzy values were used in [11] and numeric values were used in [13] and [17]) and then define the impact of the event on current goals. In [11], the authors measure this impact through fuzzy values. In [17] the impact is the difference of the probability of goal attainment after and before the event occurrence. A matrix of event impacts on goals were used by [13] where impacts are computed using a recursive goal attainment method.

Based on these approaches, we have build up a quite different method to assess the desirability of an event. In our approach, we consider only top-level goals (sub-goals are not considered). Some initial predefined importance values (weights/priorities) that may change over time due to the physical, situational and social factors described above, are assigned to a civilian goals. For each goal, we have defined the events that may supply/hamper its attainment. We distinguish between positive and negative events. Table II illustrates an example of the events that may impact negatively the goal 'Keep safe' and those that have a positive impact on this goal. We assume that only one goal is active for each civilian. The impact of the event is then measured for the current goal. In our context, the impact of the event on the agent goal depends only on the importance of the goal and the intensity of the event. For example, the impact of the 'Injury (self)' event depends on the importance of the current goal and on the degree of the injury measured through the current health state. In order to measure the intensity of an event, we define an event intensity scale composed of five equal levels in $[0,1]$. When an event takes place, we compute its intensity level EIL (Event Intensity Level). Note that the intensity level of an event may change over time. Therefore, it is recomputed periodically. The EIL is used to compute the Impact $(e, g)$ of an event $e$ on the agent goal $g$. A high value of EIL means a strong impact of the event on the goal, and conversely. The impact may be positive or negative depending on the valence of the event Valence $(e)$ in $\{-1,1\}$. The Impact $(e, g)$ is then a value in $[-1,1](1)$.

$$
\operatorname{Impact}(e, g)=\operatorname{EIL}(e) \times \operatorname{Valence}(e)
$$

The Desirability of an event $e$ at time $t$, Des $(e, t)$, is 
calculated based on the importance of the current goal $g$ Importance $(g)$ and on Impact $(e, g)(2)$. The result is a value that belongs to $[-1,1]$.

$$
\operatorname{Des}(e, t)=\operatorname{Importance}(g) \times \operatorname{Impact}(e, g)
$$

TABLE II: EVENTS INFLUENCING THE 'KEEP SAFE' GOAL

\begin{tabular}{lll}
\hline \hline \multirow{2}{*}{ Goal } & $\begin{array}{l}\text { Events with negative } \\
\text { impact on the goal }\end{array}$ & $\begin{array}{l}\text { Events with positive impact } \\
\text { on the goal }\end{array}$ \\
\hline \multirow{2}{*}{ Keep safe } & $\begin{array}{l}\text { InDanger (self) } \\
\text { Injury (self) }\end{array}$ & $\begin{array}{l}\text { ManageRisk (element) } \\
\text { Rescue (self) }\end{array}$ \\
\hline
\end{tabular}

Well-being emotions represent reactions to the Events related to a civilian. The emotion Joy (respectively Distress) arises with an intensity IJoy, (respectively IDistress ) if a desirable (respectively undesirable) event having the desirability $\operatorname{Des}(e, t)$ happens at time $t$ to the civilian (3).

$$
\begin{aligned}
& I J o y=\operatorname{Des}(e, t) \\
& \text { IDistress }=|\operatorname{Des}(e, t)|
\end{aligned}
$$

\section{b) Fortune-of-Others emotions}

These emotions appear when the appraised event concerns what happens to other people (Related to other civilians). This category of emotions is modeled in few computational models of emotions such as [10], [11] and [13]. The evaluation of the events related to others is performed using the DesirabilityForOther and Liking variables.

The Desirability for Others (DesirabilityForOther) of an event computation requires at least a partial model of the other's goals [5]. To assess this desirability, we endow the civilians with a cognitive empathy mechanism. The agent can then simulate mentally the state of the other with its current goal to compute the desirability of the event for other. We consider that what is desirable for others is desirable for self and what is undesirable for the others is undesirable for self. Therefore, we consider only what is called empathetic emotions in the OCC model (HappyFor and SorryFor).

The Liking degree between two agents is used to compute empathetic emotion oriented to the others. We assign predefined liking values between agents. This necessitates a specification of social relations. We assume that there are three degrees of social relations: family member, authority and other. A civilian considers that its family members have the highest liking degree $(=1)$. It has a lower liking degree $(=2 / 3)$ with authorities, that are rescuers (Actors). Finally, it considers that the others have the lowest liking degree $(=1 / 3)$.

A civilian $c_{i}$ is HappyFor (respectively SorryFor) a civilian $c_{j}$ if a desirable (respectively undesirable) event $e$ with the desirability DesForOther $(e, t)$ for $c_{i}$ happens at time $t$ to $c_{j}$. The civilian $c_{i}$ has a liking degree Liking $\left(c_{i}, c_{j}\right)$ to $c_{j}$. The intensities IHappyFor and ISorryFor of these emotions are computed as follows:

$$
\begin{aligned}
& I_{\text {HappyFor }}\left(c_{i}, c_{j}\right)=(\alpha \text { DesForOther }(e, t)) \times\left(\beta \operatorname{Liking}\left(c_{i}, c_{j}\right)\right) \\
& I_{\text {SorryFor }}\left(c_{i}, c_{j}\right)=(\alpha \mid \text { DesForOther }(e, t) \mid) \times\left(\beta \operatorname{Liking}\left(c_{i}, c_{j}\right)\right)
\end{aligned}
$$

\section{c) Prospect-based emotions}

When the current state of a civilian requires the intervention of other persons, he can expect various events.
Prospect based emotions arise when the events related to the civilian expectations are appraised. This category of events is generally appraised through the following appraisal variables: Desirability and Likelihood and/or expectedness (or Expectation) like in [10], [11] and [13]. In our model we have used a different set of appraisal variables, that we judge necessary in an emergency situation, to compute the intensities of prospect-based emotions.

The Likelihood of a prospected event corresponds to its probability to take place given the agent current state. For example, the probability of being helped augments if the agent health state is stable or if there is a rescuer near him. The likelihood of an event to occur corresponds to the probability of the event to happen given the current state of the civilian and the existence of a helper.

The Effort is the resource investment degree of a person in order to achieve a goal or to avoid a situation [5]. The effort is measured through the number of tries that the civilian has made to provoke an event in order to change its current state (number of calls for help when injured, number of tries to escape a risky place). We compute the number of tries that an agent has made to change a given state. We suppose that an agent have up to ten possibilities to react to the situation he is facing. After that, this value is reinitialized to zero. The number of tries is then scaled down in order to obtain a value in $[0,1]$ useful in emotion intensities computation.

The Urgency variable defines the necessity of a rapid reaction to a situation [8]. We consider that the urgency is related to the current physical state of the civilian. For example, the urgency of being rescued is greater when the health state of an agent attains a certain minimum threshold. The current health state value is divided by the value of the heath state of a safe person. The resulting value is in $[0,1]$. The Realization variable is used in order to indicate if a prospected event happens (Realization $=1$ ) or doesn't take place $($ Realization $=(-1))$.

The emotion Hope (respectively Fear) arises with an intensity IHope (respectively IFear ) if a positive (respectively negative) event is expected. The intensities of these emotions depend on the desirability of the expected event e $(\operatorname{Des}(e, t))$, the urgency of the occurrence of that event $(\operatorname{Urg}(e, t))$, the effort made to make it happen $(\operatorname{Effort}(e, t))$ and its likelihood $(L H(e, t))$ to take place at time $t(5)$. The hope degree decreases when the urgency or effort increases and increases if the likelihood increases. However, the fear degree augments when the urgency and effort augment and diminushes when the likelihood augments.

$$
\begin{aligned}
& \text { IHope }=\operatorname{Des}(e, t) \times(1-\gamma \operatorname{Urg}(e, t) \times \operatorname{Effort}(e, t)) \times \lambda L H(e, t) \\
& I F e a r=|\operatorname{Des}(e, t)| \times \gamma \operatorname{Urg}(e, t) \times \operatorname{Effort}(e, t) \times(1-\lambda L H(e, t))
\end{aligned}
$$

When an expected positive (respectively negative) event is realized (Realization $=1$ ), the result is a Satisfaction (respectively Fear-confirmed) emotion. However, if the positive (respectively negative) event doesn't happen (Realization $=(-1)$ ), the Disappointment (respectively Relief) emotion arises.

\section{2) Action appraisal}

Actions are evaluated using the Praiseworthiness appraisal variable. The evaluation of agent action results on the 
apparition of attribution emotions (Standard-based emotions). As declared above, we actually consider only one standard that is helping a human in need, which is a Praiseworthy action. Consequently, ignoring to help a person in need is a Blameworthy action. Attribution emotions are modeled in [11], [13] and [17] and are ignored in [10]. In [11], the agent learns the praiseworthiness of actions. Works presented in [13] and [17] are based on this approach to evaluate the praiseworthiness of actions. In our case, the praiseworthiness of actions could not be learned. This is due to the fact that we are modeling emotions in realistic human like agents and not reproducing emotion mechanisms on non human artificial agents such as the approaches described above.

\section{a) The civilian is the actor}

The Pride (respectively Shame) emotion arises if the civilian performs a praiseworthy (respectively a blameworthy) action towards others. The degree of the action praiseworthiness depends on the capacity of the agent to perform the action. In fact, a healthy person perceiving a danger may prefer to escape the danger to helping an injured civilian. If he chooses to escape the danger, the sense of shame will be attenuated by the existence of a risk that prohibits the help action. We define a Risk $(t)$ as a negative event that may alter the health state of a human if not escaped at time $t$. The intensity of the risk corresponds actually to the EIL (e) defined above (6). We intend in the future to add the effect of the personality on the assessment of the risk.

$$
\operatorname{Risk}(t)=\operatorname{EIL}(e)
$$

Equation (7) is used to measure the praiseworthiness or blameworthiness of an action $a$ at time $t$ (Praiseworthy $(a, t)$ or Blameworthy $(a, t))$.

$$
\begin{aligned}
& \text { Praiseworthy }(a, t)=1-\operatorname{Risk}(t) \\
& \text { Blameworthy }(a, t)=\operatorname{Risk}(t)-1
\end{aligned}
$$

The subsequent formulas (8) are used to calculate the intensities of the emotions Pride and Shame:

$$
\begin{aligned}
& \text { IPride }=\text { Praisew orthy }(a, t) \\
& \text { Ishame }=\mid \text { Blameworthy }(a, t) \mid
\end{aligned}
$$

\section{b) Another civilian is the actor}

A civilian may be in a situation that may necessitate the help of other civilians. If the expected action is realized then an emotion will arise consequently towards that civilian. Admiration (respectively Reproach) arises if another civilian performed the expected praiseworthy (respectively a blameworthy) action. In an emergency situation, the Admiration and Reproach degrees depend on how much the person needs to be helped. If this need augments, then the intensity of the emotion decreases and conversely. This need is measured through the current Health State Level of the civilian $c$ at time $t$ (HSL $(c, t))$ that is scaled down to correspond to a value in $[0,1]$. This value is used to measure the praiseworthiness (PraiseworthyOther $(a, t)$ ) of the help action and the blameworthiness (BlameworthyOther $(a, t)$ ) of not to help action of another civilian (8).

$$
\begin{aligned}
& \text { PraiseworthyOther }(a, t)=1-\operatorname{HSL}(c, t) \\
& \text { BlameworthyOther }(a, t)=\operatorname{HSL}(c, t)-1
\end{aligned}
$$

The intensities of the Admiration and Reproach emotions of a civilian $c_{i}$ for a civilian $c_{j}$ after the action of $c_{j} a\left(c_{j}\right)$ are computed in (9).

$$
\begin{aligned}
& \operatorname{IAdmiration}(c i, c j)=\text { PraiseworthyOther }(a(c j), t) \\
& \operatorname{IReproach}(c i, c j)=\mid \text { BlameworthyOther }(a(c j), t) \mid
\end{aligned}
$$

\section{3) Object appraisal}

These emotions appear as a result of an object appraisal. This kind of emotions is rarely modeled in a normal context, since objects may not always have significance to persons. However, in a crisis environment, the aspects of objects are important to the agents. These objects are evaluated using two variables: Appealingness and Familiarity. The objects that exist in the environment (buildings, roads, etc.) may catch the attention of the civilian positively (a hospital) or negatively (a blocked road). The Appealingness of an object $o$ at time $t$ (Appealingness $(o, t)$ ) corresponds to the alteration degree of its normal aspects. An object $o$ becomes more familiar (Familiarity $(o, t)$ ) to the civilian when they are frequently perceived in the environment. An agent may like (respectively dislike) an object $o$ at time $t$ with an intensity ILike (respectively IDislike ) (10). The perception of special categories of buildings such as refuges or hospitals may contribute to the realization of goals such as the 'Keep safe' or the 'Get saved' goals.

$$
\begin{aligned}
& \text { ILike }=\text { Appealingness }(o, t) \times(1-\text { Familiarity }(o, t)) \\
& \text { IDislike }=\mid \text { Appealingness }(o, t) \mid \times(1-\text { Familiarity }(o, t))
\end{aligned}
$$

\section{Emotions Thresholds and Emotional State}

The result of the emotion generation process is the emotion $E$ felt by the individual. The manifestation of an emotion needs the definition of an activation threshold. The latter is the value above which the emotion is elicited. Different emotion thresholds are defined depending on the civilian personality. The Emotional State (ES) of a civilian corresponds to the set of all emotions triggered by events and actions that take place in the encounter of a civilian. Each emotion $\mathrm{Em}_{\mathrm{i}}$ is represented by a vector that contains the category $C$, the valence $V$ and the intensity $I$ and the Target $T$. The emotional state of a civilian at a time step $t$ of the simulation is a set of $n$ vectors representing the $n$ emotions felt by the individual (11). Emotions may be altered due to new events or actions. An emotion update function is therefore used to rebuild the emotional state of the civilian.

$$
E S(t)=\left(\begin{array}{l}
E_{1}\left(C_{1}, V_{1}, I_{1}, T_{1}\right) \\
E_{2}\left(C_{2}, V_{1}, I_{2}, T_{n}\right) \\
\cdots \\
E_{n}\left(C_{n}, V_{n}, I_{n}, T_{n}\right)
\end{array}\right)
$$

\section{E. Decay Function}

After a certain period of time, the intensities of emotions diminish. We defined therefore a decay function to manage 
the decrease of emotions intensities over time. This function computes the intensity of an emotion $i$ at time $t\left(I \_E_{i}(t)\right)$ given its intensity at time $(t-1)\left(I_{-} E_{i}(t-1)\right)$ and its decay rate decRate (12).

$$
\forall_{i}, I \_E_{i}(t)=I \_E_{i}(t-1) \times\left(I \_E_{i}(t-1)\right)^{\text {decRate }}
$$

We defined different decrease rates depending on the emotion nature. For example, negative emotions duration is superior to positive emotions duration. This rate is also related to personality.

\section{CONCLUSION AND FUTURE WORK}

In this paper we have proposed an emotion generation computational model for the simulation of human emotional dynamics in emergency situations. We have provided first a generic modeling of a disaster space during an emergency situation. The generation of emotions is then computed basing-on this model and using the OCC model. The final goal is to build an empathic civilian agent based simulator in a crisis context. This simulator will be assessed via its integration in the RoboCupRescue (RCR) simulation system. Indeed, the RCR system is a virtual world for the study and the simulation of natural disasters [18]. Its purpose is to explore new methods for crisis management. The current civilian agents used in RCR are characterized by a limited set of capabilities and an extremely simple human behavior [19]. We propose therefore to improve the realism of the civilian simulation in RCR with a heterogeneous population, where individuals have different personalities and emotions. The simulation of a realistic behavior is achieved through empathic responses and interactions based on emotional dynamics. The development of these new civilian agents will allow us to test and to validate our modeling efforts during the different stages of our researches. The modeling choices we have performed aim to not alter the overall performance of the RCR simulation system. In fact, the proposed model will serve to implement the emotion generation process in each civilian agent. Therefore, the computation mechanism is distributed. Moreover, the formula used to compute appraisal variables and emotion intensities are not resource consuming. The implementation of the proposed computational model of emotion generation for the civilian agents is the next step of our research. After that, we intend to model and to implement the effect of emotions on individual and collective behaviors. We also aim to study the interpersonal emotional dynamics through emotional contagion in an emergency situation.

\section{REFERENCES}

[1] L. Luo, Z. Suiping, C. Wentong, L. Michael, L. Malcolm, H. Yoke, and S. Kabilen, "HumDPM: A decision process model for modeling human-like behaviors in time-critical and uncertain situations," Lect. Notes Comput. Sci., vol. 6670, pp. 206-230, 2011.

[2] M. H. Zaharia, F. Leon, C. Pal, G. Pagu, N. Baykara, and N. Mastorakis, "Agent-Based simulation of crowd evacuation behavior," in Proc. 2009 WSEAS Conf., 2009, pp. 529-533.

[3] U. Valentin, F. Coudret, E. Gouardères, and W. Lefe, "Modélisation du comportement humain pour la simulation d'évacuation de bâtiment en feu," Rev. Électronique Francoph. d'Informatique Graph., vol. 5, no. 2, pp. 41-51, 2011.

[4] H. Aydt, M. Lees, L. Luo, W. Cai, M. Y. H. Low, and S. K. Kadirvelen, "A computational model of emotions for agent-based crowds in serious games," in Proc. 2011 IEEE/WIC/ACM Conf., 2011, vol. 2, pp. 72-80.
[5] A. Ortony, G. L. Clore, and A. Collins, The Cognitive Structure of Emotions, Cambridge University Press, 1988.

[6] A. Damasio, Descartes' Error: Emotion, Reason, and the Human Brain, New York, USA, 1994

[7] A. Sloman and M. Croucher, "Why robots will have emotions," in International Proc. the 7th Joint Conference on Artificial intelligenc, vol. 1, San Francisco: Morgan Kaufmann Publishers Inc. , 1981, pp. $1-10$.

[8] K. R. Scherer, "Appraisal considered as a process of multilevel sequential checking," in Appraisal Processes in Emotion Theory Methods, Research, vol. 92, K. R. Scherer, A. Schorr, and T. Johnstone, Eds. Oxford University Press, 2001, pp. 92-120.

[9] R. S. Lazarus and C. A. Smith, "Emotion and adaptation," in Handbook of Personality: Theory and research, L. A. Pervin, Ed. Guilford, New York, 1990, pp. 609-637.

[10] J. Gratch, S. Marsella, P. Petta, and S. C. Marsella, "EMA: A process model of appraisal dynamics," Cogn. Syst. Res., vol. 10, no. 1, pp. 70-90, 2009.

[11] M. S. E. Nasr, J. Yen, and T. R. Ioerger, "FLAME-fuzzy logic adaptive model of emotions," Auton. Agent. Multi. Agent. Syst., vol. 3, no. 3, pp. 219-257, Sep. 2000.

[12] J. Dias and A. Paiva, "Feeling and reasoning: A computational model for emotional characters," Prog. Artif. Intell., vol. 3808, pp. 127-140, 2005.

[13] M. Kazemifard, N. G. Aghaee, and T. I. Ören, "Design and implementation of GEmA: A generic emotional agent," Expert Syst. Appl., vol. 38, no. 3, pp. 2640-2652, 2011.

[14] F. Kebair and F. Serin, "Towards a multiagent decision support system for crisis management," Journal of Intell. Syst., vol. 20, no. 1, pp 47-60, March 2011.

[15] F. Kebair, "Modélisation multiagent de postes de commande coordonnateurs de prise de décisions stratégiques: application au système de simulation de RoboCupRescue," Ph.D. dissertation, Dept. Computer science, Havre Univ., Havre, FR, 2009.

[16] J. Broekens and D. DeGroot, "Scalable and flexible appraisal models for virtual agents," in Proc.2004 Game-on Conf., 2004, pp. 208-215.

[17] B. T. Duy, "Creating emotions and facial expressions for embodied agents," Ph.D. dissertation, Centre of telematics and Info. Tech Twente Univ., Enschede, AE, 2004.

[18] RoboCupRescue official web site. [Online]. Available: http://www.robocup.org/robocup-rescue/.

[19] A. Khorsandian and A. Abdolmaleki, "RoboCupRescue 2009 - Rescue Simulation League ( Infrastructure Competition ) Team Description MRL2009 - brave circles ( Iran ),” 2009.

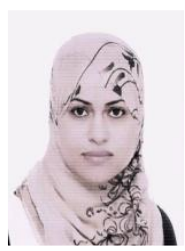

M. Belhaj has received her B.S. degree and M.S. degree in computer science applied to management from the Higher Institute of Management (ISG) of Tunis, University of Tunis, Tunisia. She is actually a Ph.D. student at the ISG of Tunis, University of Tunis. She is also a member of the Optimization Strategies and Intelligent Information Engineering Laboratory (SOIE Lab, Tunisia). Her research focuses on artificial intelligence, emotions, multi-agent Systems, simulation and modeling.

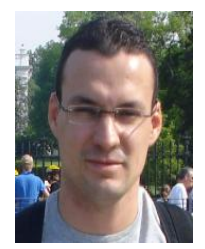

F. Kebair has received the B.S. degree in computer science applied to management from the ISG of Tunis, University of Tunis, Tunisia in 2004. He has obtained the M.S. degree and the Ph.D. degree in computer science from the University of Le Havre, France, in 2005 and 2009 respectively. Dr. Kebair is currently an associate professor at the Higher Institute of Computer Science of Tunis (ISI, Tunisia). He is also member of the Optimization Strategies and Intelligent Information Engineering Laboratory (SOIE Lab, Tunisia). His research interests cover essentially multi-agents systems, simulation, decision support systems.

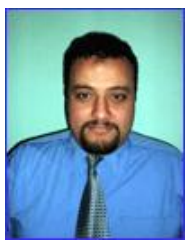

L. Ben Said has received the B.S. degree in computer science applied to management from the ISG of Tunis, University of Tunis, Tunisia in 1998. He has obtained the M.S. degree and the Ph.D. degree in computer science from the University of Paris VI, France, in 1999 and 2003 respectively. He was research fellow for three years at France Telecom (R\&D). Dr. Ben Said is actually an associate professor at the Higher Institute of Management of Tunis (ISG, Tunisia). He is also now the head of the Optimization Strategies and Intelligent Information Engineering Laboratory (SOIE Lab, Tunisia). His research interests cover essentially multi-agents systems, simulation, multi-criteria decision making, evolutionary computation and behavioral economics. 